\title{
LAS CONCEPCIONES DEL MEDIOAMBIENTE EN ESTUDIANTES DE NIVEL SUPERIOR
}

Elizabeth González Urda

Docente del Ciclo Básico Común de la Universidad de Buenos Aires, Argentina

\section{INTRODUCCIÓN}

Es mayoritariamente aceptado que los alumnos no son una página en blanco sobre la cual imprimir el conocimiento del docente, por lo tanto, sus concepciones previas son una información fundamental a la hora de planificar la enseñanza. Sobre esta base, es que decidimos indagar cómo los alumnos de nivel superior conciben al medioambiente.

El medioambiente es un concepto complejo y el estudio de su didáctica es sumamente interesante y enriquecedor ya que se trata del concepto base de la educación ambiental. Al involucrar conocimientos de diferentes disciplinas (Brailovsky, A y Foguelman D, 1991; Leff, 2000), este concepto presenta una complejidad con implicancias directas en su enseñanza.

La concepción del medioambiente ha seguido una historia de cambios: desde la idea más simple, aquello que rodea al hombre o a una población biológica cualquiera-, hasta la noción más actual y compleja que lo concibe como un sistema resultante de la interacción entre sistemas sociales y naturales. (Vigil,1994; Meinardi y col, 2002; Funtowicz y De Marchi, 2000). En esta posición, las sociedades son las que interactúan con el medio circundante, las que determinan las condiciones de intervención sobre lo natural, las que a lo largo de sus historias han modificado su relación con el medio circundante. En concreto, no es el hombre como ser individual ni como ser vivo el que hace a la noción de medioambiente, sino las sociedades como subsistemas con sus elementos y relaciones propios. Según el tema o problema que se analice, la escala de la sociedad a considerar puede ser desde un pequeño poblado hasta la humanidad toda, pero siempre entendiendo que este componente del concepto de medioambiente constituye también un sistema.

Es precisamente esta concepción de medioambiente la que mejor se relaciona con la educación ambiental ya que la incorporación de la sociedad permite identificar interacciones de doble vía: las sociedades usan recursos e intervienen sobre la naturaleza y al mismo tiempo el mundo natural condiciona, provee y responde a las intervenciones humanas.

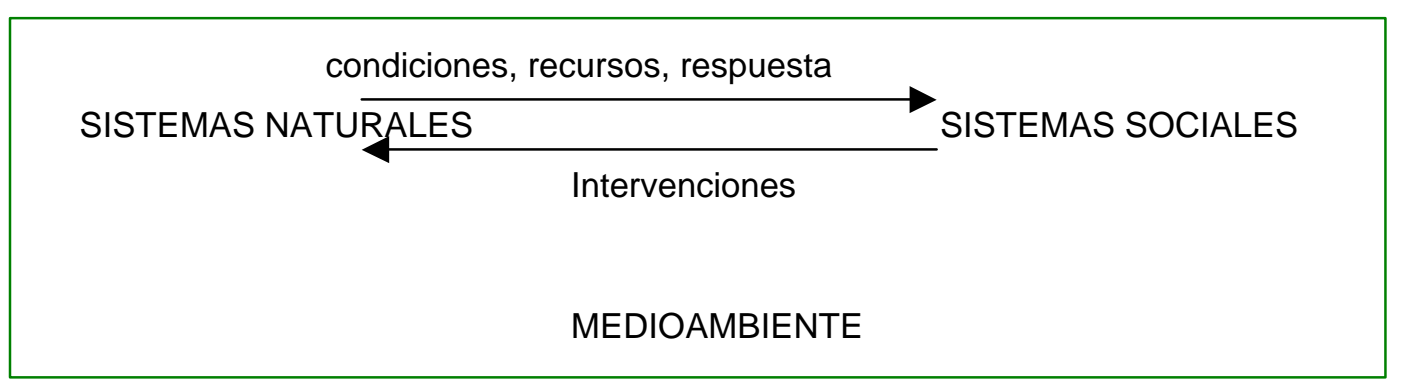

La compresión de este sistema complejo requiere entonces de diferentes disciplinas: ecología, química, geografía, economía, sociología, historia, etc. La sola mención de estas áreas de conocimiento pone en evidencia las dificultades que entraña su abordaje en la educación formal como, por ejemplo: 
- la formación marcadamente disciplinar de los docentes. La curricula de los institutos de formación docente que continúa con las líneas disciplinares de las universidades. Es decir, licenciaturas o profesorados de ciencias naturales totalmente inconexos con los de ciencias sociales.

- la inserción compartimentalizada en el curriculum. En general, los equipos técnicos que diseñan la curricula escolar están formados por expertos que repiten la lógica interna de sus propias disciplinas,

- la polisemia del término. Los términos ambiente o medioambiente están insertos en el lenguaje cotidiano. Las cuestiones ambientales llegan a nuestros alumnos y docentes, no sólo desde el ámbito académico sino también desde los medios de comunicación social. Un rápido análisis sobre el uso de estos términos indica que se aplican a nociones diferentes: desde las condiciones meteorológicas o las habitaciones de una casa a casos de interacción naturalezasociedad. Sumado a esto, en algunos ámbitos, ambos términos se aplican indistintamente y, en otros, hay un rechazo al uso del vocablo medioambiente por considerarlo redundante 0 argumentando que el ambiente no es la mitad de algo. Estas últimas posturas son, en general, defendidas por grupos ecologistas que están insertos en la defensa social y política de las cuestiones ambientales y tiene más llegada a los medios de difusión social que los expertos disciplinares.

\section{METODOLOGIA DE TRABAJO}

Todo lo expuesto, hace que nos resulte muy atractivo investigar la enseñanza de este concepto.

Si bien se han realizado algunos estudios acerca del tratamiento pedagógico de conceptos ambientales complejos (Rojero, 2000; Luffiego y Rabadán, 2000; Leff, 2000) y de la identificación de concepciones de medio (Astolfi,1999) y medioambiente (Loughland y col, 2002.), en éstos no se ha considerado el nivel de la educación superior.

El objetivo de este trabajo fue relevar las concepciones de ambiente y medioambiente que tienen los alumnos de carreras ambientales de nivel superior con el fin de, en el futuro, diseñar estrategias didácticas que atiendan a estos resultados. Seleccionamos esta población porque se trata de alumnos que una vez egresados actuarán en el campo profesional técnico, segmento de la sociedad tradicionalmente marcado por la sectorización disciplinar y donde estimamos es fundamental incorporar la mirada interdisciplinar en el abordaje de las cuestiones ambientales que deberán resolver en su ejercicio profesional. Para ello se indagó, el primer día de clases, en dos años sucesivos, a alumnos pertenecientes al primer y segundo año de la carrera de Técnico Superior en Ecología de un Centro Educativo de Nivel Terciario y de segundo año de la carrera de Licenciatura en Ciencias del Ambiente de una universidad, ambos de la ciudad de Buenos Aires. A dos de los grupos se les solicitó que respondieran qué era ambiente. A otro grupo se lo interrogó acerca de qué era ambiente o medio ambiente y si creían que había diferencias entre los dos términos las explicitaran, por último a un cuarto grupo se le hizo las dos preguntas: qué es medioambiente y qué es ambiente. 
Se obtuvieron 92 respuestas que fueron analizadas según una versión ampliada de la clasificación propuesta por Loughland y col. (2002) y el análisis epistemológico de la noción de medio realizado por J.P. Astolfi, (1988). Se ordenaron las respuestas en categorías con un sentido creciente de complejidad conceptual, desde una visión globalizada hacia una concepción sistémica del medioambiente. Las tres primeras están focalizadas en el medioambiente como lugar, ya sea indiferenciado o que contiene cosas. En las cuatro últimas, las relaciones entre elementos son el rasgo distintivo.

En nuestro caso se consideró la oportunidad de incorporar otra categoría (Categoría 4), - intermedia entre las dos focalizaciones propuestas por Loughland y col.-, que reúne las respuestas donde se explicita la relación entre elementos. Esta ampliación de categorías se fundamentó en la necesidad de indagar la importancia que los alumnos pudieran darle en sus definiciones a las relaciones entre elementos.

En las otras tres categorías siguientes, las relaciones son unidireccionales (Categorías 5 y 6) o bidireccionales (categoría 7) Las posibles concepciones y sus características son:

\begin{tabular}{|c|c|c|c|}
\hline Concepción & & Característica & Frase tipo \\
\hline $\begin{array}{l}\text { Focalizadas en el } \\
\text { objeto: }\end{array}$ & & & \\
\hline 1 & $\begin{array}{l}\text { El medioambiente es } \\
\text { un lugar }\end{array}$ & $\begin{array}{lr}\text { Concepción } & \\
\text { indiferenciada, } & \\
\text { globalizada, } & \\
\text { precientífica } & \text { o } \\
\text { enumeración } & \text { de } \\
\text { elementos inertes } & 0 \\
\text { condiciones. } & \\
\end{array}$ & $\begin{array}{lr}\text { Todo lo que } & \text { nos } \\
\text { rodea./Espacio } & \text { físico } \\
\text { que nos } & \text { rodea./ } \\
\text { Conjunto } & \text { de } \\
\text { condiciones climáticas. }\end{array}$ \\
\hline 2 & $\begin{array}{l}\text { El medioambiente es } \\
\text { un lugar que contiene } \\
\text { cosas vivas. }\end{array}$ & $\begin{array}{l}\text { Concepción mecánica, } \\
\text { analítica, aditiva. }\end{array}$ & $\begin{array}{l}\text { Es el espacio donde } \\
\text { todas las especies } \\
\text { viven./ Es el lugar } \\
\text { donde habitan o son } \\
\text { contenidos los } \\
\text { ecosistemas. / Espacio } \\
\text { en el cual se desarrolla } \\
\text { un individuo. }\end{array}$ \\
\hline 3 & $\begin{array}{l}\text { El medioambiente es } \\
\text { un lugar que contiene } \\
\text { cosas vivas y gente }\end{array}$ & $\begin{array}{l}\text { Concepción mecánica, } \\
\text { analítica, aditiva que } \\
\text { incorpora al hombre } \\
\text { como si fuera cualquier } \\
\text { otra especie. }\end{array}$ & $\begin{array}{l}\text { Es el espacio físico en } \\
\text { el cual compartimos los } \\
\text { recursos todos los } \\
\text { seres vivos. }\end{array}$ \\
\hline \multicolumn{4}{|l|}{ Intermedia: } \\
\hline 4 & $\begin{array}{l}\text { El medioambiente es } \\
\text { un lugar donde } \\
\text { interactúan elementos } \\
\text { vivos e inertes }\end{array}$ & $\begin{array}{l}\text { Concepción mecánica, } \\
\text { analítica, sin mención } \\
\text { de lo social pero } \\
\text { explicitandor la } \\
\text { posibilidad r de } \\
\text { interacción entre lo vivo } \\
\text { y lo inerte }\end{array}$ & $\begin{array}{l}\text { Es el lugar o habitat } \\
\text { donde conviven } \\
\text { algunos seres bióticos } \\
\text { y abióticos que se } \\
\text { relacionan entre sí. }\end{array}$ \\
\hline \multicolumn{4}{|l|}{$\begin{array}{l}\text { Focalizadas en las } \\
\text { relaciones }\end{array}$} \\
\hline 5 & $\begin{array}{l}\text { El medioambiente hace } \\
\text { cosas por la gente. }\end{array}$ & $\begin{array}{l}\text { Concepción mecánica, } \\
\text { analítica. El ambiente } \\
\text { puede ayudar a la } \\
\text { calidad de vida. El } \\
\text { sentido de la } \\
\text { interacción es del } \\
\text { medioambiente hacia } \\
\text { las sociedades }\end{array}$ & $\begin{array}{l}\text { Es el conjunto de } \\
\text { recursos naturales que } \\
\text { hacen posible la } \\
\text { supervivencia humana } \\
\text { y de todo ser vivo. }\end{array}$ \\
\hline
\end{tabular}




\begin{tabular}{|c|c|c|c|}
\hline & & humanas. & \\
\hline 6 & $\begin{array}{ll}\text { La gente } & \text { es } \\
\text { responsable por } & \text { el } \\
\text { medioambiente. }\end{array}$ & $\begin{array}{l}\text { El foco es lo que las } \\
\text { sociedades pueden } \\
\text { hacer por el ambiente. } \\
\text { La interacción es } \\
\text { personal o de grupos } \\
\text { reducidos y en un solo } \\
\text { sentido }\end{array}$ & $\begin{array}{l}\text { Es el lugar donde } \\
\text { estamos viviendo./No } \\
\text { tires papeles, hacelo } \\
\text { por el medioambiente } \\
\text { para cuidar el ambiente } \\
\text { en que estamos. }\end{array}$ \\
\hline 7 & $\begin{array}{l}\text { El medioambiente es el } \\
\text { resultado de la } \\
\text { interacción entre los } \\
\text { sistemas sociales y los } \\
\text { naturales }\end{array}$ & $\begin{array}{llr}\text { Integración } & & \text { de } \\
\text { sistemas social } & \text { y } \\
\text { natural. } & & \text { El } \\
\text { medioambiente o } & \text { el } \\
\text { ambiente no } & \text { son } \\
\text { externos na a la } \\
\text { sociedad. Interacción } \\
\text { de doble vía. } & \end{array}$ & $\begin{array}{l}\text { Es el resultado de la } \\
\text { interacción entre los } \\
\text { sistemas sociales y } \\
\text { naturales }\end{array}$ \\
\hline
\end{tabular}

\section{RESULTADOS OBTENIDOS}

En todos los grupos de alumnos analizados las respuestas mayoritarias corresponden a la primera y segunda categoría, concepción que se mantiene aún en alumnos que han cursado un año de carreras ambientales. Por otra parte, subyace una idea de exterioridad, el ambiente como una entidad que es externa a los seres vivos y en muchos caso difícil de caracterizar a juzgar por las respuestas de tipo global como "Es todo lo que nos rodea". Los bajos porcentajes alcanzados por las concepciones donde se priorizan las relaciones (5\% a $32 \%$, según los grupos) indicarían la dificultad de concebir un concepto basado en interacciones.

Entre el 4,65\% y el 33,33\%, según los grupos de alumnos, no respondieron o indicaron no acordarse la definición. Por otra parte, algunas respuestas no pudieron ser clasificadas ya que focalizaban en otras cuestiones, por ejemplo: "Ambiente es el medio donde se hace un estudio", "Organización natural o no de los seres vivos".

En el grupo 4, donde se solicitó a los alumnos que definieran tanto ambiente como medioambiente, el $22 \%$ de los alumnos indicaron que eran sinónimos. En aquellos casos en que establecieron diferencias, se compararon las dos respuestas y se observó que lo social, cuando aparece, está asociado a la noción de medioambiente y con una relación unidireccional (La naturaleza provee recursos para el hombre. Concepción 5). En las definiciones de ambiente lo humano no aparece.

De los datos obtenidos se observa que a pesar del nivel educativo analizado; sólo aproximadamente el $1 \%$ de los alumnos elabora una definición compleja de medioambiente. Entre un $14 \%$ y $44 \%$, según los grupos, tienen una visión indiferenciada, mientras que entre el $33 \%$ y el $52 \%$ identifican componentes, pero no establecen relaciones entre ellos.

Las respuestas de categoría 6 y 7 sólo se dieron en un grupo (Grupo 3) sumando alrededor de un $10 \%$ de las respuestas.

Se presentaron varias respuestas donde se cuestionó el uso del término medioambiente indicando que el ambiente no era la mitad de nada o que el uso de este término no era correcto por ser redundante. Los datos obtenidos indican que estos cuestionamientos de índole semántica llegan hasta la educación formal distrayendo el análisis del concepto en sí. 


\section{CONCLUSIONES}

En los estudiantes de carreras ambientales de nivel superior cuyas respuestas fueron analizadas, se observa que, tanto al ingresar como después de un año de carrera, sus ideas de medioambiente o ambiente están mayoritariamente centradas en la idea de lugar o sitio físico. Los aspectos relacionales o de interacción aparecen en la minoría de las respuestas a pesar de ser el núcleo de la actual concepción de medioambiente.

La relación entre los sistemas sociales y los sistemas naturales fue considerada en sólo una de las 96 respuestas analizadas. La interacción más frecuente entre estos dos componentes fue en una única vía: los sistemas naturales son proveedores del hombre y en una única respuesta se indicó al hombre como responsable de los cambios ambientales.

Esta dificultad para concebir un concepto basado en relaciones nos cuestiona como docentes acerca de, por ejemplo, el aprendizaje del concepto de ecosistema, que al igual que el concepto de medioambiente va más allá de la simple suma de factores bióticos y abióticos. Si la mayoría de estos alumnos indagados han aprobado las materias introductorias a la ecología de sus respectivas carreras resulta preocupante que sostengan una concepción de medioambiente de carácter aditivo con escasa mención de relaciones.

En un momento en que lo ambiental llega a los alumnos de todos los niveles desde las fuentes más variadas (la televisión, los periódicos, los textos escolares, etc.) resulta importante plantearse de qué estamos hablando cuando mencionamos al medioambiente o al ambiente y replantear la práctica docente. Dado que en los alumnos coexisten diferentes concepciones de muy distinto grado de complejidad, esta heterogeneidad debería ser puesta de manifiesto antes de trabajar cualquier tema ambiental, ya que la comprensión de la complejidad del concepto actual de medioambiente permitiría interpretar las cuestiones ambientales cotidianas, aceptar el aporte de las diferentes disciplinas e integrarlo en una unidad común.

Varias podrían ser las propuestas para trabajar en el aula el concepto de medioambiente de modo sistémico:

- Análisis de casos con identificación de actores sociales y posturas frente al problema.

- Audiencias públicas simuladas sobre casos de actualidad.

- Proyectos ambientales escolares en los que se expliciten los factores naturales, sociales y sus relaciones.

- Crítica de los textos escolares incorporando los aspectos sociales o naturales cuando estén ausentes.

- Análisis del tratamiento de las cuestiones ambientales y el uso de los términos específicos en artículos periodísticos o presentaciones en los medios de difusión social, etc.

Si los alumnos no conciben a la sociedad de la que forman parte como integrante del medioambiente, será más difícil que puedan involucrarse y actuar. En este caso, el logro de los objetivos de la educación ambiental internacionalmente aceptados, (sensibilidad, conciencia, responsabilidad crítica, valores sociales, participación, etc.) y permanentemente declamados será mucho más difícil. 


\section{BIBLIOGRAFÍA}

ASTOLFI, Jean Pierre. El aprendizaje de conceptos científicos: aspectos epistemológicos, cognitivos y lingüísticos. Enseñanza de las ciencias, 6 (2), 1998, 147-155 PP.

ASTOLFI, Jean Pierre. El "error", un medio para enseñar. Colección: investigación y enseñanza, n 15. Sevilla, Editora Díada, 1999.

BRAILOVSKY, Antonio y FOGUELMAN, Dina. Memoria verde. Historia ecológica de la Argentina. Buenos Aires, Ed. Sudamericana, 1991.

FUNTOWICZ, Silvio y DE MARCHI, Bruna. Ciencia posnormal, complejidad reflexiva y sustentabilidad. En Leff, Enrique (Coord.) La complejidad ambiental. Ed Siglo XXI México, 2000.

LEFF, Enrique. Epistemología ambiental. San Pablo, Cortez Editora. 2000. LOUGHLAND Tony, REID, Anna y PETOCZ, Peter. Young People's Concepcions of Enviroment:A Phenomenographic Analysis. Enviromental Education Research. Vol 8 ( 2), 2002, 187-197. h

LUFFIEGO GARCIA, Máximo y RABADÁN VERGARA, José. Evolución del concepto de sostenibilidad y su introducción en la enseñanza. Enseñanza de las ciencias 18 (3): 2000. 473-486 pp.

MEINARDI, Elsa, ADÚRIZ-BRAVO, Agustín y REVEL CHION, Andrea, La educación ambiental en el aula. Una propuesta para integrar contenidos multidisciplinares a través de la argumentación. Investigación en la escuela 46 (Monográfico de abril) 2002.

VIGIL, Carlos A. Aproximación a la problemática ambiental. Elementos para su análisis. Buenos Aires. Ed. Biblos, 1994. 